\title{
Profile of Single Mode Fiber Coupler Combining with Bragg Grating
}

\author{
Romi Fadli Syahputra ${ }^{1}$, Saktioto ${ }^{{ }^{2}}$, Ros Meri $^{3}$, Syamsudhuha ${ }^{4}$, Okfalisa $^{5}$ \\ ${ }_{1,2,3}$ Physics Dept., FMIPA, Universitas Riau, Pekanbaru, Indonesia \\ ${ }^{4}$ Department of Mathematics, Faculty of Math and Natural Sciences, Universitas Riau, Indonesia \\ ${ }^{5}$ Department of Information Technique, Faculty of Science and Technology, \\ Universitas Islam Negeri SUSKA, Indonesia \\ *Corresponding author: saktioto@yahoo.com
}

\begin{abstract}
This paper describes a numerical experiment of design and operation of a fiber coupler between single mode fiber and fiber Bragg grating (FBG). Both components are coupled depending with optical waveguide and source parameters. A characterization of fiber coupler is simulated by varying long grating of $10 \mathrm{~mm}$ to $60 \mathrm{~mm}$ using transfer matrix method based on coupled mode equation. The wave peak, transmission, and dispersion parameters are analyzed to determine the performance of the fiber coupler. The transmission spectrum showed the wave peaks rise to any increase in the grating length on channel 1 and channel 2. Transmission on channel 1 and channel 2 decreased from the wavelength range of $1.45 \mu \mathrm{m}-1.55 \mu \mathrm{m}$ and rised in the range of $1,55 \mu \mathrm{m}-1,65 \mu \mathrm{m}$ for each increment in length of grating. The dispersion showed the zero dispersion at specific wavelength for each increase in length of grating. This component can be applied for controlling information signal in wide range communication.
\end{abstract}

Keywords: Fiber coupler, Fiber Bragg grating (FBG), Transmission spectrum

Copyright $\odot 2017$ Universitas Ahmad Dahlan. All rights reserved.

\section{Introduction}

Although the fiber component has been successfully applied and produced commercially, the development of fiber designs and components keep continuing to investigate to achieve more applicable and multifunction in communication system such as fiber coupler and Fiber Bragg Grating (FBG). Fiber coupler is a waveguide that is composed of two parallel optical fibers capable of transmitting the channels, known as Multiplexing. Besides it can also serve as a beam splitter and a power divider. Currently fiber coupler is a device of optical communication which is being developed as an optical sensor considering the advantages possessed by these devices, among others: it has a large bandwidth with attenuation of small power, small size and lightweight, and resistant to electromagnetic induction [1-3]. Optical fiber as the sensor can be applied in various physical measurement parameters such as friction, temperature, pressure, humidity, fluid flow rate, the rate of rotation, the concentration of a substance, as well as chemical analysis [3]. Experiments of optical fiber as sensor-based shift in intensity modulation have been performed by utilizing coupling losses of fiber optic using LED sources. It has also been performed experiments using multi-mode and single mode fiber with good results but it is high coupling losses. Another method to lower the losses it performed using a directional coupler optical fiber with single mode based phase modulation mode that produces high sensitivity but low reach $[4,5]$.

Fiber coupler is basically an optical fiber core of each fiber is created adjacent to the coupling region specific length using fusion techniques $[3,6]$. Light entered on one fiber will experience the exchange of power from one fiber to another fiber periodically and exit at the end of each fiber output with a division of power that depend on the coupling ratio. The analysis used in this component generally uses the theory coupling modes that can be resolved with the transfer matrix method. Completion of the initial coupling mode equation theory is seeking coupling coefficient that determines power can move to other waveguides.

Currently, the fabrication of fiber coupler is more complicated and requires fairly expensive equipment, while fiber coupler is needed in a variety of applications in the field of optical sensors. Because of that, this study is conducted to design and simulate the fiber 
coupler composed of a combination of single mode fiber and FBG. FBG is an optical fiber mode single section of the core fiber made a variation of the refractive index periodically distributed in the form of segmentation resembles the grating and can reflect certain wavelengths and transmit the remainder, so that the FBG can serve as reflector optics or filter optics.

Based on the characteristics possessed by the fiber Bragg grating and a coupler, both combinations are designed on one type of constituent fiber grating by varying the length of 10 $\mathrm{mm}, 20 \mathrm{~mm}$, and $30 \mathrm{~mm}$. This study is conducted to determine the effect of long-grating against the wave peak, transmission and fiber dispersion at the optical sensor components. The combination of the FBG fiber coupler provides a time delay which makes the optical sensor can provide more accurate information.

\section{Research Methodology}

The method begins with a basic equation of coupling mode equations that are solved by a transfer matrix method. The transfer matrix Equation [7] is given by:

$$
T_{i}=\left|\begin{array}{cc}
\cosh \left(\gamma l_{i}\right)-i \frac{\Delta \beta}{\gamma} \sinh \left(\gamma l_{i}\right) & -\frac{\kappa}{\gamma} \sinh \left(\gamma l_{i}\right) \\
i \frac{\kappa}{\gamma} \sinh \left(\gamma l_{i}\right) & \cosh \left(\gamma l_{i}\right)+i \frac{\Delta \beta}{\gamma} \sinh \left(\gamma l_{i}\right)
\end{array}\right|
$$

Where $\Delta \beta$ is the wave vector detuning with the formulation of $\Delta \beta=\beta-\pi / \Lambda$, where $\beta$ is the propagation constant of the fiber core which can be calculated by $\beta=2 \pi n_{\text {eff }} / \lambda$ and $\gamma=\sqrt{\kappa^{2}-\Delta \beta^{2}}$. The coupling coefficient $\kappa$ in z-axes can be calculated by the following equation:

$$
\kappa(z)=\frac{\pi \Delta n_{e f f} u g(z)}{\lambda}
$$

For uniform FBG, $\Delta n_{\text {eff }}$ is constant with a function $g(z)=1$ and the number of rings for $u=1$ so that the Equation 2 can be written as:

$$
\kappa=\frac{\pi \Delta n_{e f f}}{\lambda}
$$

The transmission spectrum is obtained by inputting parameters of component of fiber coupler. Fiber 1 and 2 use a single mode fiber with a core value of the radius and cladding $4.1 \mu \mathrm{m}$ and $62.5 \mu \mathrm{m}$ respectively corresponding to the cladding radius and the refractive index of the core and coating of 1.4682 and 1.4615 . Fiber 1 inserted FBG with period $2 \mathrm{~mm}$, with the modulation of the refractive index of $6 \times 10^{-4}$, the number of grating is 25 , as well as long-grating varied from $10 \mathrm{~mm}, 20 \mathrm{~mm}, 30 \mathrm{~mm}, 40 \mathrm{~mm}, 50 \mathrm{~mm}$, and $60 \mathrm{~mm}$. The fiber coupler design with combination to FBG is depicted in Figure 1.
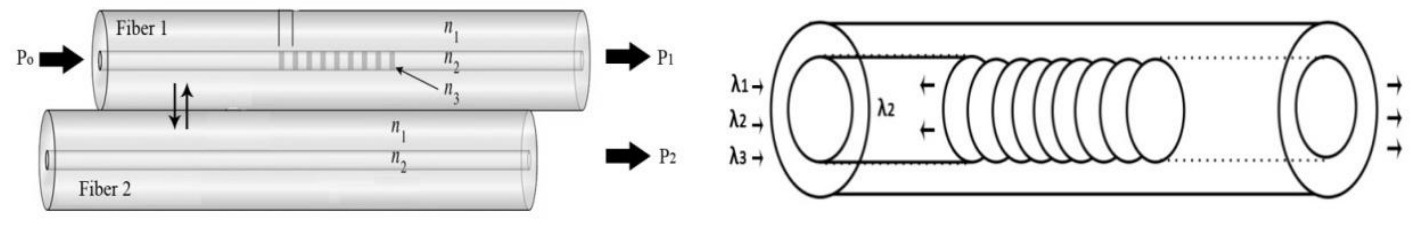

Figure 1. Power propagation of fiber;

The leaf side is shown power propagation for input power $P_{\circ}$ and output power $P_{1}$ and $P_{2}$; The right side is depicted schematic design of FBG [8] 


\section{Result and Analysis}

The transmission spectrums are obtained by varying the length of FBG in order to investigate the peak of a wave, transmission, and dispersion as described in Table 1.

Table 1. Transmission of peak wave varied on grating length

\begin{tabular}{llll}
\hline No. & $\begin{array}{l}\text { Grating Length } \\
(\mathrm{mm})\end{array}$ & $\begin{array}{l}\text { Wave Peak } \\
(\mu \mathrm{m})\end{array}$ & $\begin{array}{l}\text { Transmission } \\
(\mathrm{dB})\end{array}$ \\
\hline 1. & 10 & 1.47372 & -23.3137 \\
2. & 20 & 1.52136 & -28.6281 \\
3. & 30 & 1.53934 & -39.1006 \\
4. & 40 & 1.54888 & -44.8535 \\
5. & 50 & 1.55480 & -35.3143 \\
6. & 60 & 1.60432 & -21.8653 \\
\hline
\end{tabular}

Table 1 describes channel 1, the increment of length of grating shows the peak of the transmission spectrum bandwidth has increased generally. This condition is caused by increasing the length of grating on the fiber core resulting in a long disturbance area that will be passed by the transmission spectrum. The greater area disorder results in the transmission spectrum will experience greater power losses, so that the transmitted energy will be smaller and the peak of the transmission spectrum will experience widening. Widening the greater peak transmission makes the transmitted power fiber will be smaller, so the transmission value obtained will be smaller for each increment of grating length. Another condition is shown by the length of grating $50 \mathrm{~mm}$ and $60 \mathrm{~mm}$ increase the transmission value from the previous one. Shifting the peak of the transmission spectrum waves occur due to the material dispersion that occurs in the core of the fiber. Spectrum with higher frequencies will have greater power losses; this is due to greater volumes of power absorbed by the fiber core material in this wavelength range, so that the peak of the transmission spectrum will continue to shift in the spectrum with a lower frequency. These conditions explain the reason for the peak shift of the transmission spectrum at wavelengths greater for each increase in the grating length which is also found in electromagnetic device, such as radar [9].

The use of infrared as light sources in optical fibers is divided in multiple windows spectrum. Window spectrum with a wavelength of $1.300 \mu \mathrm{m}-1.550 \mu \mathrm{m}$ is the window that is commonly used because it gives the power losses is much smaller than the entire existing spectrum. While the grating length of $50 \mathrm{~mm}$ and $60 \mathrm{~mm}$, the peak of the transmission spectrum is at a wavelength of $1.55480 \mu \mathrm{m}$ and $1.60432 \mu \mathrm{m}$ corresponding to high infrared spectrum. This infrared wavelength, the losses of power generated will be smaller. This is due to the absorption of power by the fiber core material at wavelengths above $1.550 \mu \mathrm{m}$ is not greater than the absorption that occurs below the wavelength of $1.550 \mu \mathrm{m}$.

Table 2. Transmission values on peak wave transmission length variation 2 based on grating.

\begin{tabular}{llll}
\hline No. & $\begin{array}{l}\text { Grating Length } \\
(\mathrm{mm})\end{array}$ & $\begin{array}{l}\text { Wave Peak } \\
(\mu \mathrm{m})\end{array}$ & Transmission $(\mathrm{dB})$ \\
\hline 1. & 10 & 1.57076 & -14.2172 \\
2. & 20 & 1.57528 & -14.2820 \\
3. & 30 & 1.57690 & -14.3049 \\
4. & 40 & 1.61416 & $-5.86 \times 10^{-4}$ \\
5. & 50 & 1.60696 & $-5.58 \times 10^{-3}$ \\
6. & 60 & 1.60226 & $-5.05 \times 10^{-2}$ \\
\hline
\end{tabular}

Table 2 shows a decrease of transmission on the length of the grating $10 \mathrm{~mm}, 20 \mathrm{~mm}$, and $30 \mathrm{~mm}$ and then ride on the grating length of $40 \mathrm{~mm}$ then down to the grating length of 50 $\mathrm{mm}$ and then increases again at $60 \mathrm{~mm}$. The greater value of transmission two is caused by the peaks at high infrared wavelengths.

Figure 2 shows that the grating peak of transmission for two waveguide where the grating length is varied. There is peak wave shift increased. 


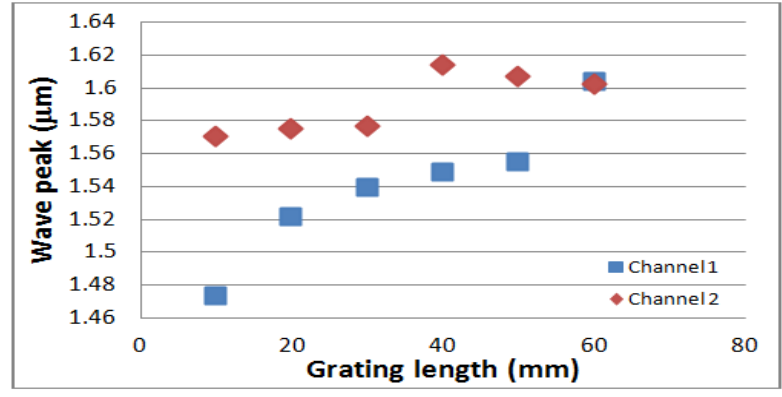

Figure 2. Peak profile on transmission wave

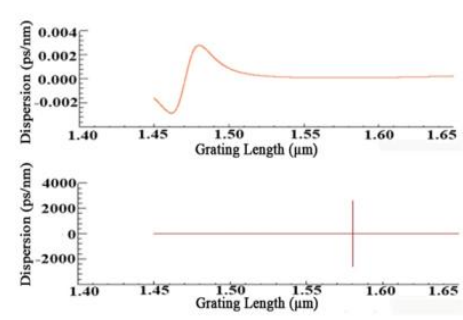

(a)
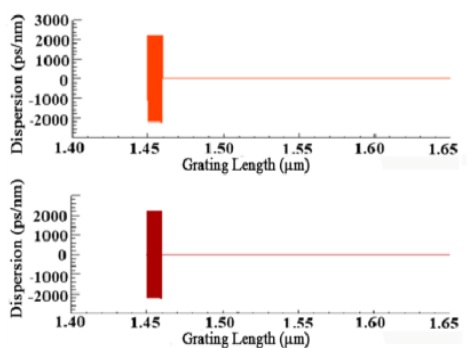

(d)

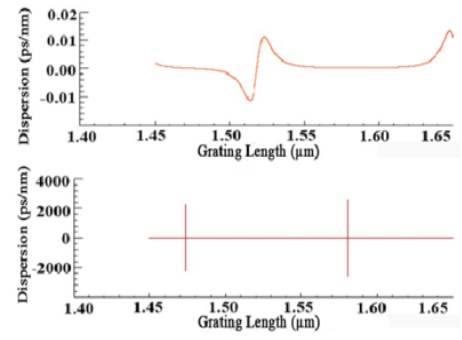

(b)
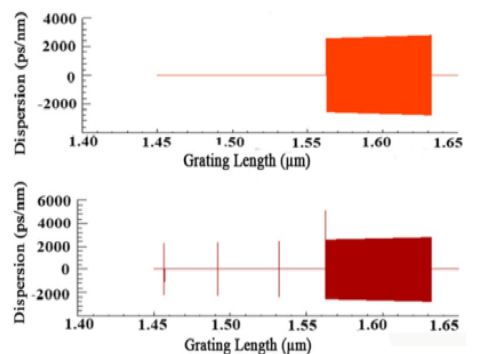

(e)

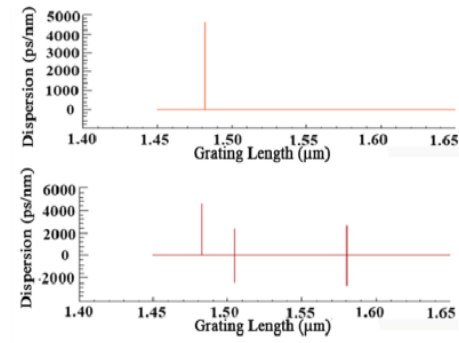

(c)
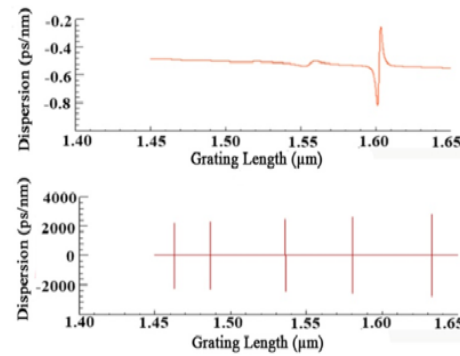

(f)

Figure 3. The dispersion of Fiber. The top is dispersion for waveguide 1 and the bottom for waveguide 2 with grating length of (a) $10 \mathrm{~mm}$, (b) $20 \mathrm{~mm}$, (c) $30 \mathrm{~mm}$, (d) $40 \mathrm{~mm}$, (e) $50 \mathrm{~mm}$, and (f) $60 \mathrm{~mm}$, respectively

The spectrum of light with a wavelength range of different incoming and propagating in the fiber core will experience different degrees of refraction. Components of different wave lengths result differences in velocity in the fiber core producing light pulses will undergo expansion as chromatic dispersion. This dispersion is a combination of material and waveguide dispersion. Chromatic dispersion that rises is not constant, but it rather has a particular dispersion value at any wavelength and will reach zero dispersion value at a specific wave length. The grating length used in varying the refractive index of the fiber core on channel 1 is a part of the material dispersion, so it provides a variety of chromatic dispersion that occurs for any variation in the grating length.

In Figure 3 on channel 1, for each additional length of grating given provides the shift peak dispersion at different wavelengths. In the infrared wavelength of $1.450 \mu \mathrm{m}-1.550 \mu \mathrm{m}$, the peak of dispersion decreased for each increment in length of grating given to approach zero dispersion. The wavelength range of $1.550 \mu \mathrm{m}-1.650 \mu \mathrm{m}$, the zero dispersion value occurred at $50 \mathrm{~mm}$. On channel 2, wavelength with zero dispersion increases for each increment in grating length. Based on propagation signal, this FBG and fiber coupling can be applied to control the power signal in wide range communication system. 


\section{Conclusion}

The design simulation has successfully performed on fiber coupler for single mode fiber and FBG component. The wider the length of the grating, the crest of the wave transmission will shift to the larger wavelengths where the peak value of the wave on each grating length respectively - i.e channel 1: $1.47372 \mu \mathrm{m}, 1.52136 \mu \mathrm{m}, 1.53934 \mu \mathrm{m}, 1.54888 \mu \mathrm{m}, 1.55480 \mu \mathrm{m}$, $1.60432 \mu \mathrm{m}$ and channel 2: $1.57076 \mu \mathrm{m}, 1.57528 \mu \mathrm{m}, 1.57690 \mu \mathrm{m}, 1.61416 \mu \mathrm{m}, 1.60696 \mu \mathrm{m}$ and $1.60226 \mu \mathrm{m}$. The wider of grating length, the value transmission on channel 1 and 2 will be smaller in the peak range of $1.45 \mu \mathrm{m}-1.55 \mu \mathrm{m}$ and transmission value will rise again in the range of $1.55 \mu \mathrm{m}-1.65 \mu \mathrm{m}$. The resulting dispersion is not constant, but rather provides value of a particular dispersion at any wavelength, and will reach a value of zero dispersion wave length specified for each increment of grating length. The resulting response time is longer so as to provide the greater accuracy on detection in application. By designing the FBG and fiber coupler the optical power signal can be applied for wide range of telecommunication system.

\section{Acknowledgements}

The authors thank the Ministry of Research, Technology and Directorate General of Higher Education, Indonesia through research grant of Simlitabmas Ristek Dikti, for generous support of this research in 2017.

\section{References}

[1] Barrias A, Casas JR, Villalba S. A Review of Distributed Optical Fiber Sensors for Civil Engineering Applications. Sensors. 2016; 16(5): 1-35.

[2] Fidanboylu K, Efendioğlu HS. Fiber Optic Sensors and Their Applications. Proceeding of $5^{\text {th }}$ International Advanced Technologies Symposium. Karabuk. 2009: 1-6.

[3] Khare RP. Fiber Optics and Optoelectronics. First Edition. New Delhi: Oxford University Press. 2004: 323-345.

[4] Lee SH, Darmawan S, Lee CW, Chin MK. Transformation between Directional Couplers and Multimode Interferometers based on Ridge Waveguides. Optics Express. 2004; 12(14): 3079-3085.

[5] Wu DKC, Lee KJ, Pureur V, Kuhlmey BT. Performance of Refractive Index Sensors Based On Directional Couplers in Photonic Crystal Fibers. Journal of Lightwave Technology. 2013; 31(22): 3500-3510.

[6] Senior JM. Optical Fiber Communications Principles and Practice. Harlow: Pearson Education Limited. 2009: 256-280.

[7] Ikhlef A, Hedara R, Chikh-Bled M. Uniform Fiber Bragg Grating Modelling and Simulation Used Matrix Transfer Method. International Journal of Computer Science Issues. 2012; 9(2): 368-374.

[8] Sharma R, Rohilla R, Sharma M, Manjunath TC. Design \& Simulation of Optical Fiber Bragg Grating Pressure Sensor for Minimum Attenuation Criteria. Journal of Theoretical and Applied Information Technology. 2009; 5(5): 515-530.

[9] Rocca P, Morabito AF. Optimal synthesis of reconfigurable planar arrays with simplified architectures for monopulse radar applications. IEEE Transaction on Antennas Propagation. 2015; 63(3):10481058. 